\title{
The Cohomology of the Groups of Order 32
}

\author{
By David J. Rusin
}

\begin{abstract}
We have calculated the mod-2 cohomology rings of all the groups of 32 elements. This paper describes the methods of calculation; the computer routines used can be adapted to assist in the calculation of the modular cohomology rings of other finite groups. We also describe the results of the calculations; the data we have collected provide a substantial increase in the supply of completed calculations in group cohomology, and so we take this opportunity to compare known results and open conjectures.
\end{abstract}

This project may be viewed as the penance done for a youthful boast: that one can always calculate the (mod-2) cohomology of any single (finite 2-) group if one is patient enough. We report here the results of the computations for the 51 groups of order 32 .

For general background material on group cohomology, one may consult [8]. It should be remarked that the definition of $H^{n}\left(G, \mathbf{F}_{2}\right)$ lends itself to an easy if inefficient algorithm to compute this abelian group; but it is hopeless to compute the entire ring $H^{*}(G)=\bigoplus_{n} H^{n}\left(G, \mathbf{F}_{2}\right)$ in this way. By various techniques, the cohomology rings of the groups of order at most 16 have been determined (see the last column of the tables in Section 3), but these groups exhibit little of the variety observed in the groups of order 32 .

Roughly half of the text of this paper (Section 1) is devoted to the techniques of computation. We describe the algorithms used in generality that may suffice to compute the cohomology of larger groups. Most of the computations were, in fact, done originally by hand, but the strain on our patience was greatly relieved with the introduction of Stillman and Bayer's computer program Macaulay, which can compute syzygies over polynomial rings; many computational problems can be reduced to this one.

In Section 2 we describe the results of these computations. We have commented on several conjectures and areas of research for which this new crop of data has proven helpful.

Finally, in a long appendix (Section 3) we provide the complete data for our groups.

1. Description of the Algorithms. The computations break naturally into several stages.

(1.1) Identification of the Groups. The groups of order 32 (and 64) are classified in [7]. The abelian groups are listed by their invariants in ascending order (e.g., "(2)(3)" for $\mathbb{Z}_{4} \times \mathbb{Z}_{8}$ ). Each nonabelian group carries a name such as $32 \Gamma_{7} a_{3}$ which identifies it as a member $\left(a_{3}\right)$ of order 32 in a certain family $\left(\Gamma_{7}\right)$. The eight families

Received October 21, 1987.

1980 Mathematics Subject Classification (1985 Revision). Primary 18-04, 20J06, 57T35. 
of groups of order 32 are distinguished by their central quotients $\bar{G}=G / Z(G)$ (where $Z(G)$ and $Z G$ denote the center of $G$ ); these quotients are

$$
1, \mathbb{Z}_{2}^{2}, D_{8}, \mathbb{Z}_{2}^{3}, \mathbb{Z}_{2}^{4}, D_{8} \times \mathbb{Z}_{2}, 16 \Gamma_{2} c_{1}, D_{16}
$$

respectively for $\Gamma_{1}, \Gamma_{2}, \ldots, \Gamma_{8}\left(D_{n}\right.$ is the dihedral group of order $\left.n\right)$.

Thus, for each $G$ we have an exact sequence

$$
1 \rightarrow Z(G) \stackrel{i}{\rightarrow} G \stackrel{\pi}{\rightarrow} G / Z(G) \rightarrow 1
$$

with well-understood ends. The three groups in $(*)$ are presented in [7] with generators and relations in a way that makes the maps $i$ and $\pi$ clear.

We remark that, in general, the subdivision of these groups into families is only a fair predictor of the structure of the cohomology rings.

(1.2) Selection of Spectral Sequence. We choose to approximate $H^{*}(G)$ using the Eilenberg-Moore spectral sequence (EMSS) of the extension $\left(^{*}\right)$, as was done in [12] (see that paper for more details on the technique). In particular, we do not use the Serre spectral sequence: it converges more slowly, it leaves undecided some higher differentials, and it requires the use of different rings $E_{r}$ at each stage of the computation. By contrast, we observe the EMSS collapses at $E_{2}$ for almost every group.

We also observe that for most groups $G$ there are other central extensions than $\left(^{*}\right)$ to which the EMSS may be applied. For a specific $G$ it is probably better to mod out by a smaller central subgroup, since then some structure of $H^{*}(G)$ can be inflated from $H^{*}(\bar{G})$. The disadvantage is that the $E_{2}$ term will be correspondingly more complex. Moreover, since we are computing the cohomology of many groups, we find it convenient to minimize the number of quotients $\bar{G}$ to consider.

The EMSS begins with the term

$$
E_{2}=\operatorname{Tor}_{H^{*}\left(K(Z G, 2), \mathrm{F}_{2}\right)}\left(H^{*}(G / Z G), \mathbf{F}_{2}\right),
$$

where $K(Z G, 2)$ is an Eilenberg-MacLane space associated to the center $Z G$ of the group $G$ (see [12]). It is straightforward to compute this ring, once certain pieces of data are given. If, for example, $Z(G)=\mathbb{Z}_{2}$, then the base ring $R=$ $H^{*}\left(K(Z G, 2), \mathbf{F}_{2}\right)$ is a polynomial ring $\mathbf{F}_{2}\left[t_{0}, t_{1}, t_{2}, \ldots\right]$ in infinitely many variables, with $t_{n}=S q_{1}\left(t_{n-1}\right)$. The structure of $H^{*}(\bar{G})$ as a module over this is determined by the map $f: B \bar{G} \rightarrow K(Z G, 2)$ implicit in $\left(^{*}\right): f^{*}\left(t_{0}\right) \in H^{2}(\bar{G})$ is the extension cocycle, $f^{*}\left(t_{1}\right)=S q^{1}\left(f^{*}\left(t_{0}\right)\right), f^{*}\left(t_{2}\right)=S q^{2}\left(f^{*}\left(t_{1}\right)\right)$, and so on.

If $Z G$ is cyclic of larger order, the same remarks apply except that $t_{1} \neq S q^{1}\left(t_{0}\right)$. If $Z G=A_{1} \times A_{2}$ is noncyclic, we note

$$
H^{*}\left(K(Z G, 2), \mathrm{F}_{2}\right)=H^{*}\left(K\left(A_{1}, 2\right)\right) \otimes H^{*}\left(K\left(A_{2}, 2\right)\right)
$$

is again a polynomial ring, and the algebra structure is again determined by the various $f^{*}\left(t_{n}^{(j)}\right)$. We make concrete these remarks in several steps.

(1.3) Calculation of Extension Cocycles. We need to know the fundamental classes $q_{j}=f^{*}\left(t_{0}^{(j)}\right) \in H^{2}(\bar{G})$, and for cyclic factors of $Z G$ of order greater than 2 , the classes $\beta_{j}=f^{*}\left(t_{1}^{(j)}\right) \in H^{3}(\bar{G})$. 
LEMMA A. (1) If $Z(G)=C_{1} \times C_{2} \times \cdots$ with $C_{j}$ cyclic, let $N_{j}=\prod_{i \neq j} C_{i}$. Then the fundamental classes $q_{j}$ and $\beta_{j}$ are the extension cocycles of

$$
1 \rightarrow Z G / N_{j} \rightarrow G / N_{j} \rightarrow \bar{G} \rightarrow 1
$$

in $H^{2}(\bar{G})$ and $H^{3}(\bar{G})$, respectively.

(2) If $Z G$ is cyclic and $\Phi(Z G)$ its Frattini subgroup, then $q \in H^{2}(\bar{G})$ is also the extension cocycle of the extension

$$
1 \rightarrow Z G / \Phi(Z G) \rightarrow G / \Phi(Z G) \rightarrow \bar{G} \rightarrow 1
$$

(3) If $Z G$ is cyclic and $Z G \leq H \leq G$, then the fundamental classes $q^{\prime} \in$ $H^{2}(H / Z G)$ and $\beta^{\prime} \in H^{3}(H / Z G)$ of the extension

$$
1 \rightarrow Z(G) \rightarrow H \rightarrow H / Z(G) \rightarrow 1
$$

are the restrictions of $q, \beta \in H^{*}(\bar{G})$.

The proofs amount to a diagram chase. Part (3) is actually quite useful: there are often one or more subgroups $H<G$ for which $q^{\prime}$ and $\beta^{\prime}$ are easily determined; together they may pin down $q$ and $\beta$ unambiguously. This happens if the kernels of the restriction maps $\operatorname{Res}_{H / Z G}^{\bar{G}}$ meet $H^{2}(\bar{G})$ or $H^{3}(\bar{G})$ only in (0); we then say these $H$ detect $H^{2}(\bar{G})$ or $H^{3}(\bar{G})$. We will rely frequently on detection in this paper.

LEMMA B. (1) If $\bar{G}=G / Z(G)$ for any group $G$ in families $\Gamma_{1}, \ldots, \Gamma_{6}$ or $\Gamma_{8}$, then $H^{*}(\bar{G})$ is detected by the elementary (-abelian) subgroups of $\bar{G}$. If $G \in \Gamma_{7}$, this is true of $H^{2}(\bar{G})$ only.

(2) If $\bar{G}$ is elementary-abelian, then for $n \leq 3, H^{n}(\bar{G})$ is detected by the subgroups of order 4 .

The proof is a direct calculation.

If $G$ is a group of 32 in family $\Gamma_{7}, Z G$ is of order 2. Thus Lemmas A and B imply that for any group $G$ of order 32 the fundamental classes we need are all determined by their restrictions to elementary-abelian subgroups of $\bar{G}$ of order 4 . By Lemma A(1) these, in turn, are the fundamental classes of extensions in which the central subgroup is cyclic and the quotient is $\mathbb{Z}_{2}^{2}$. Thus, we are now down to calculating the following fundamental classes:

LEMMA C. Let

$$
1 \rightarrow \mathbb{Z}_{n} \rightarrow H \rightarrow \mathbb{Z}_{2}^{2} \rightarrow 1
$$

be a central extension, with $\mathbb{Z}_{n}$ any cyclic group. Let the fundamental invariants be $q, \beta \in H^{*}\left(\mathbb{Z}_{2}^{2}\right)$. Write $\bar{H}$ for $H / \mathbb{Z}_{n}$.

(1) If $H$ is quaternion (of order 8), then $q=x^{2}+y^{2}+x y$ and $\beta=x y(x+y)$, where $x$ and $y$ are any generators of $H^{1}\left(\mathbb{Z}_{2}^{2}\right)$.

(2) If $H$ is dihedral (of order 8), let $x, y \in H^{1}(\bar{H})=\operatorname{Hom}\left(H, F_{2}\right)$ have noncyclic kernels. Then $q=x y$ and $\beta=x y(x+y)$.

(3) If $H$ otherwise has a cyclic subgroup $M$ of index 2 , let $x \in H^{1}(\bar{H})$ have kernel $M$. Then $q=x^{2}$. If $H$ is abelian, $\beta=0$; and if not, $\beta=x y(x+y)$ where $y \in H^{1}(\bar{H})$ is linearly independent of $x$. 
(4) In the remaining cases, $q=0$. If $H$ is abelian, $\beta=0$ too; if not, $\beta=$ $x y(x+y)$, where $x$ and $y$ generate $H^{1}(\bar{H})$.

Thus, armed with the restriction maps, we compute the $q_{j}$ and $\beta_{j}$ for all our extensions.

(1.4) The $E_{1} T e r m$. We construct the higher invariants from the preceding ones: $f^{*}\left(t_{n}^{(j)}\right)=S q_{1}\left(f^{*}\left(t_{n-1}^{(j)}\right)\right)$, where $S q_{1}: H^{*}(\bar{G}) \rightarrow H^{*}(\bar{G})$ may be calculated easily. If

$$
S q: H^{*}(\bar{G}) \rightarrow H^{*}(\bar{G})[T]
$$

is the total squaring operation, $S q=S q_{0}+T S q_{1}+T^{2} S q_{2} \ldots$, then $S q$ is a ring homomorphism and so is determined by its action on generators; this is all known for our 8 groups $\bar{G}$. We can then compute

$$
S q_{1}(x)=\left.\frac{d}{d T}(S q(x))\right|_{T=0}
$$

in Macaulay, and so calculate all $f^{*}\left(t_{n}^{(j)}\right)$.

As $H^{*}(\bar{G})$ is Noetherian, these all lie in a finitely generated ideal, and so for $n$ sufficiently large we can express these invariants as $H^{*}(\bar{G})$-linear combinations of the preceding ones.

Now, $H^{*}(K(Z G, 2))$ is a polynomial ring in the $t_{n}^{(j)}$, so a projective resolution of the trivial module $\mathbf{F}_{2}$ is the bigraded ring

$$
E_{0}=H^{*}(K(Z G, 2)) \otimes \operatorname{Exterior}\left(\left\{u_{n}^{(j)}: n \geq 0\right\}\right)
$$

with differential $d_{0}: E_{0} \rightarrow E_{0}$ a derivation determined by $d\left(u_{n}^{(j)}\right)=t_{n}^{(j)}$. We tensor to get our complex

$$
E_{1}=H^{*}(\bar{G}) \otimes \operatorname{Exterior}\left(\left\{u_{n}^{(j)}\right\}\right)
$$

with $d_{1}: E_{1} \rightarrow E_{1}$ also a derivation, and $d\left(u_{n}^{(j)}\right)=f^{*}\left(t_{n}^{(j)}\right)$.

We need to make this a finite problem. Using the Noetherian property above, we may alter $u_{n}^{(j)}$ (with $n$ large) by some linear combination of the $u_{m}^{(j)}$ (with $m<n$ ) to get elements $\tilde{u}_{n}^{(j)}$ for which we still have

$$
E_{1}=H^{*}(\bar{G}) \otimes \operatorname{Exterior}\left(\left\{\tilde{u}_{n}^{(j)}\right\}\right),
$$

but now we have $\tilde{u}_{n}^{(j)} \in \operatorname{Ker}(d)$ for $n$ large. These $\tilde{u}_{n}^{(j)}$ then lie in $E_{2}^{-1, *}$ and hence survive to $E_{\infty}$ and represent elements of $H^{*}(G)$. (In fact, if $\tilde{u}_{n}^{(j)}$ represents some $T_{j} \in H^{*}(G)$, then $T_{j}^{2}$ will be represented by $u_{n+1}^{(j)}+$ lower-order terms.) Thus, the $E_{1}$ term is a finitely generated exterior algebra (hence module) over a ring extension $H^{*}(\bar{G})\left[T_{1}, T_{2}, \ldots, T_{r}\right]$ (where $r$ is the rank of $Z G$ ).

(1.5) The $E_{2}$ Term. This is simply the homology of the preceding complex, and so is an easy task for Macaulay to handle. We incorporate the discussion of (1.4) above and simply take $E_{0}$ as a resolution over a finitely generated polynomial ring in place of $H^{*}(K(Z G, 2))$. The program expresses $E_{2}$ as a finitely presented module over the ring $H^{*}(\bar{G})\left[T_{1}, \ldots, T_{r}\right]$. The relations it provides then approximate some of the relations used to describe $H^{*}(G)$. We will later need to solve the multiplicative extension problem and to calculate products of these module generators.

(1.6) The $E_{\infty}$ Term. Fortunately, we almost always have collapse. The $E_{2}$ terms tend to be generated, as a module over $H^{*}(\bar{G})\left[T_{1}, \ldots, T_{r}\right]$, by classes in 
$E_{\infty}^{-1, *}$. These classes of course live to $E_{\infty}$, and so all of $E_{2}$ does. In two cases, $\Gamma_{2} f$ and $\Gamma_{4} d$, we need to observe $E_{2}$ is generated as an algebra by $E_{\infty}^{-1, *}$, but the same remarks apply.

There remain two cases without obvious collapse, $\Gamma_{7} a_{1}$ and $\Gamma_{7} a_{3}$, and in both cases we do indeed have $E_{2} \neq E_{3}$ (although $E_{3}=E_{\infty}$ for the same reasons as above). The group $\Gamma_{7} a_{3}$ was described in [13]; it is easy to recover the differential $d_{2}: E_{2} \rightarrow E_{2}$ from there.

Here, briefly, is the argument for $\Gamma_{7} a_{1}$. We assume a knowledge of StiefelWhitney classes (see the appendix of [1]). One constructs (by inducing) a representation $\rho: G \rightarrow O_{4}(\mathbf{R})$ whose Stiefel-Whitney class $w_{4}(\rho) \in H^{4}(G)$ restricts nontrivially to $Z(G) \cong \mathbb{Z}_{2}$. Consequently, the Serre spectral sequence collapses at $E_{4}$, and we see $w_{4}(\rho)$ injects $H^{n}(G)$ into $H^{n+4}(G)$. In particular, since there is a certain $x \in H^{1}(G)$ with a large annihilator, there must be a class $w_{4}(\rho) \cdot x \in H^{5}(G)$ with the same annihilator. Short arguments convince us $w_{4}(\rho) \cdot x$ is represented by a certain $[\alpha] \in E_{2}^{-1,6}$. Now, ann $(x)$ includes a certain $\beta \in H^{4}(G)$ inflated from $G / Z(G)$; thus $[\beta] \in E_{\infty}^{0,4}$ should annihilate $[\alpha]$, and so $[\alpha] \cdot[\beta]=0 \in E_{\infty}^{-1,10}$. Since $[\alpha] \cdot[\beta] \neq 0$ in $E_{2}$, there must be a differential onto $[\alpha][\beta]$. The only possibility is a certain map $d_{2}: E_{2}^{-2,11} \rightarrow E_{2}^{-1,10}$.

It is perhaps not necessary to stress that this reasoning is sufficient but not encouraging: one now knows to expect $E_{2} \neq E_{\infty}$ without any confidence that one can compute higher differentials in general.

At this point we have a graded version of $H^{*}(G)$ and we turn to the extension problems. We need to identify generators of $H^{*}(G)$ to accomplish this.

(1.7) Polynomial Ring Generators. We observed earlier that $H^{*}(G)$ can be viewed as a finite module over a pure polynomial extension $H^{*}(\bar{G})\left[T_{1}, \ldots, T_{r}\right]$ of $H^{*}(G / Z G)$. There is some freedom in the choice of the $T_{i}$ : they need only restrict nontrivially in the (trivial) spectral sequence of

$$
1 \rightarrow Z(G) \rightarrow Z(G) \rightarrow 1 \rightarrow 1
$$

It is easy to argue that, except for $\Gamma_{7} a_{1}$ as noted in (1.6), we need only insure $T_{i}$ restricts nontrivially in $H^{*}(Z G)$ itself. Two common techniques are to take the $T_{i}$ to be norms or Stiefel-Whitney classes. We observed no case in which the former succeeded but not the latter (although this is no longer true for groups of larger order). Indeed, in every case but one $\left(\Gamma_{3} d_{2}\right)$, there were in fact sufficient representations to create all the classes $T_{i}$. One need only find a maximal subgroup $H<G$ and a representation $\rho_{0}: H \rightarrow O_{n / 2}(\mathbf{R})$ of half the desired degree of $T_{i}$ such that $\rho_{0}$ restricts appropriately to $Z(G) \subset H$; then let $\rho=\operatorname{Ind}_{H}^{G}\left(\rho_{0}\right)$. (Typically $H$ is cyclic, dihedral, quaternion, or a product of these.)

The exceptional case $\Gamma_{3} d_{2}$ is metacyclic and so has been discussed in [12]. Should it be necessary to consider other examples of groups without sufficient representations, it will probably be better to use the method of (1.9).

(1.8) Module Generators. From the EMSS we conclude $H^{*}(G)$ is a module over $H^{*}(\bar{G})\left[T_{1}, \ldots, T_{r}\right]$ on finitely many classes $\alpha_{i} \in E_{\infty}$. We can identify most of these as transfers in the following way.

Suppose $L \in E_{\infty}^{0,1}$ annihilates one of these $\alpha$. View $L \in H^{1}(G)=\operatorname{Hom}\left(G, \mathbf{F}_{2}\right)$ and let $M=\operatorname{Ker}(L)$ ( $M$ is maximal in $G)$. Macaulay has a feature which makes 
it possible to find the Poincaré series of $H^{*}(G)$. Since the series of $H^{*}(M)$ is also known, we can compute the series of $\operatorname{ann}(L) \subset H^{*}(G)$ using the discussion in [14]. We can also compute the annihilator of $L$ in $E_{\infty}$ and its Poincaré series. If the two are equal, we conclude that given any $\beta \in E_{\infty}$ with $L \cdot \beta=0 \in E_{\infty}$, there exists a $b \in H^{*}(G)$ represented by $\beta$ such that $L \cdot b=0 \in H^{*}(G)$. Hence by [14] again, this $b$ is a corestriction. In this way we can describe some module generators of $H^{*}(G)$ unambiguously as $\alpha_{i}=\operatorname{Cor}_{M}^{G}\left(a_{i}\right)$ for certain $a_{i} \in H^{*}(M)$.

There are some exceptions to this pattern. In some cases, of course, there are no $\alpha_{i}$, that is, $H^{*}(G)$ is already generated by the image $E_{\infty}^{0, *}$ of $H^{*}(\bar{G})$ and the $T_{i}$. For some groups $\left(\Gamma_{2} b, \Gamma_{2} e_{1}, \Gamma_{2} e_{2}, \Gamma_{2} f, \Gamma_{2} g, \Gamma_{3} b\right)$ there is an $\alpha \in E_{2}^{-1,2}$ which we can show is not a transfer; but this represents an element of $H^{1}(G)=\operatorname{Hom}\left(G, \mathbf{F}_{2}\right)$ and so is well understood. In several cases $\left(\Gamma_{2} h, \Gamma_{3} c_{2}, \Gamma_{3} e, \Gamma_{4} c_{1}, \Gamma_{6} a_{2}\right)$ we obtain generators of $H^{*}(G)$ as transfers but only by using more than one subgroup. The group $\Gamma_{7} a_{1}$, discussed above, has a module generator which cannot be a transfer, but it has already been described (as $w_{4}(\rho)$ in (1.6)).

The group $\Gamma_{7} a_{3}$ leads to an $E_{\infty}$ term in which each $\alpha_{i}$ is annihilated by a certain $L \in E_{\infty}^{0,1}$. However, we discover one $\alpha \in H^{3}(G)$ is not a transfer. In this case, we choose to describe this element by giving its restrictions to maximal subgroups. This technique is actually inappropriate to this group, since there is a nonzero ideal (generated by a single $\theta \in H^{3}(G)$ ) of elements which restrict to zero on all subgroups. Thus, our $\alpha$ is not specified uniquely by this procedure. Fortunately, the relations we provide for $H^{*}(G)$ in Section 3 are not affected by the choice of $\alpha$.

Finally, in the two cases $\Gamma_{4} c_{3}$ and $\Gamma_{4} d$ we encounter generators $\alpha_{i} \in E_{\infty}$ which have no 1-dimensional annihilators in $E_{\infty}$, let alone in $H^{*}(G)$. For these groups we succeeded only by the method of this next subsection.

(1.9) The Expansion-Restriction Technique. This is best illustrated with the following example. Suppose one needed to calculate something about $H^{*}\left(\mathbb{Z}_{\mathbf{4}}\right)-$ say, about the map $S q^{1}: H^{2} \rightarrow H^{3}$. A technique often used is to restrict to subgroups whose cohomology is better known (in this case $\mathbb{Z}_{2} \subset \mathbb{Z}_{4}$ is the only choice) and to use naturality. Unfortunately, when these subgroups fail to detect cohomology, there is some ambiguity in the answer (in our case, for example, knowing $\operatorname{Res}\left(S q^{1}(x)\right)=S q^{1}(\operatorname{Res}(x))=S q^{1}\left(a^{2}\right)=0$ does not determine $S q^{1}(x)$ itself).

Yet we observe two key properties of the dihedral group $D_{8} \supset \mathbb{Z}_{4}$ : the restriction map $H^{*}\left(D_{8}\right) \rightarrow H^{*}\left(\mathbb{Z}_{4}\right)$ is onto, and the two elementary subgroups $\mathbb{Z}_{2}^{2}<D_{8}$ detect its cohomology.

Thus, for example, $H^{*}\left(D_{8}\right)=\mathbf{F}_{2}[z, y, x] / z y$, and there is a choice of the twodimensional class $x$ whose restrictions to each $H^{*}\left(\mathbb{Z}_{2}^{2}\right)=\mathbb{F}_{2}[a, b]$ may be written $a(a+b)$ in a suitable basis $\left(b=\operatorname{Res}(z+y)\right.$, and $a \in H^{1}\left(\mathbb{Z}_{2}^{2}\right)$ could be either of the other two generators). Hence $\operatorname{Res}\left(S q^{1}(x)\right)=S q^{1}(\operatorname{Res}(x))=a(a+b) \cdot b=$ $\operatorname{Res}(x \cdot(z+y))$; because of detection, $S q^{1}(x)=x \cdot(z+y)$. Since $x$ restricts to the generator $\bar{x}$ of $H^{2}\left(\mathbb{Z}_{4}\right)$, we have $S q^{1}(\bar{x})=\operatorname{Res}(x \cdot(z+y))=0$ (since $\operatorname{Res}(z)=$ $\left.\operatorname{Res}(y) \in H^{1}\left(\mathbb{Z}_{4}\right)\right)$, and so the question about $\mathbb{Z}_{4}$ is answered.

Similar techniques were employed for the groups $\Gamma_{4} c_{3}$ and $\Gamma_{4} d$. We found extensions

$$
\begin{aligned}
& 1 \rightarrow \mathbb{Z}_{2}^{2} \rightarrow \tilde{G} \rightarrow \mathbb{Z}_{2}^{4} \rightarrow 1 \\
& \cup \quad U \\
& 1 \rightarrow \mathbb{Z}_{2}^{2} \rightarrow G \rightarrow \mathbb{Z}_{2}^{3} \rightarrow 1
\end{aligned}
$$


with the same two key properties: restriction from $\tilde{G}$ to $G$ is onto (this is observed by comparing spectral sequences) and $H^{*}(\tilde{G})$ is detected by (maximal) subgroups whose cohomology is known. (Detection is checked by showing that the ideals $(L) \in H^{*}(G)$, for the various $L \in H^{1}(G)$, meet in zero. We needed to check first that the image of $(L)$ in $E_{\infty}$ was no bigger than $L \cdot E_{\infty}$. Macaulay does it all.)

Specifically, the extensions corresponded to cocycles $q=\left(q_{1}, q_{2}\right)$ with $q_{i} \in$ $H^{2}\left(\mathbf{Z}_{2}^{4}\right)$ : for the two groups $G$ we used respectively

$$
q=\left(x^{2}+y^{2}+x z+u^{2}, y^{2}+z^{2}+y z+y u\right)
$$

and

$$
q=\left(y^{2}+x z+y u, x^{2}+x z+y z+x u\right) .
$$

In each case, $G$ itself is the subgroup $\operatorname{Ker}(u)<\tilde{G}$. Detection in the first case is by 3 groups $\mathbb{Z}_{4} \times D_{8}$ (namely $\operatorname{Ker}(x), \operatorname{Ker}(z)$, and $\operatorname{Ker}(x+z)$ ) and one $\mathbb{Z}_{\mathbf{2}} \times\left(\mathbf{Z}_{\mathbf{4}} \cdot \mathbf{Z}_{\mathbf{4}}\right)$ $(=\operatorname{ker}(u+z+y))$. In the second case, we used three subgroups $\mathbf{Z}_{4} \times \mathbf{Z}_{2}^{2}$ and one $\mathbb{Z}_{2}^{4}$.

As in the dihedral example and the case $\Gamma_{7} a_{3}$, we define elements $\tilde{\alpha}_{i} \in H^{*}(\tilde{G})$ by giving their restrictions to the four good subgroups, then calculate relations and so on down there. This gives $H^{*}(\tilde{G})$ unambiguously, and we simply have $H^{*}(G) \cong H^{*}(\tilde{G}) /(u)$ (see [14]).

We also used this technique to verify some relations in $\Gamma_{3} c_{2}$, and to remove an ambiguity in the action of $S q^{4}$ on $\Gamma_{5} a_{2}$ (the amalgamated product $D_{8} \circ Q_{8}$ ). In the latter case, it was easiest to expand to a group $\left(=D_{8} \circ D_{8} \circ D_{8}\right)$ whose order was 4 times as large.

Lacking any evidence to the contrary, we might conjecture that for every group $G$ there exists a suitable "expansion" $\tilde{G}$, so that transfers and Stiefel-Whitney classes need never be used. Certainly, as a test, one might consider the groups herein whose Steenrod-algebra action is not completely resolved.

(1.10) The Multiplicative Extension Problen. For most groups, we arrive after (1.8) at the conclusion that $H^{*}(G)$ is the module over the ring $H^{*}(\bar{G})\left[T_{1}, \ldots, T_{r}\right]$ on $1 \in H^{*}(G)$ and some transfers $\alpha_{i}=\operatorname{Cor}\left(a_{i}\right)$. Naturally, it is not a free module, but one can argue that all relations correspond to relations in $E_{\infty}$. Macaulay provides these: finitely many syzygies $\sum p_{i} \alpha_{i}=0$ with $p_{i} \in H^{*}(\bar{G})$ and all $\alpha_{i}$ in a "column" $E_{\infty}^{p, *}$. We need only calculate $\sum p_{i} \operatorname{Cor}\left(a_{i}\right) \in H^{*}(G)$; the answer will represent an element in $E_{\infty}^{p+1, *}$ (which is usually $E_{\infty}^{0, *}=H^{*}(\bar{G}) /$ relations). When all $\alpha_{i}$ are transferred from a single subgoup, this is just $\operatorname{Cor}\left(\sum \operatorname{Res}\left(p_{i}\right) \cdot a_{i}\right)$. Here we inflate our knowledge of the restriction map $H^{*}(G / Z G) \rightarrow H^{*}(M / Z G)$ to calculate the term to be transferred; the result usually lands in $\inf \left(H^{*}(M / Z G)\right)$ too, so we may inflate knowledge of the transfer map as well. Exceptional cases were easily handled by ad-hoc reasoning.

There remains the transformation of $H^{*}(G)$ from an $H^{*}(\bar{G})$-module to an algebra: we need to calculate products $\alpha_{i} \alpha_{j}$ as elements in the module. Again, we use the formula $\alpha_{i} \alpha_{j}=\alpha_{i} \cdot \operatorname{Cor}\left(a_{j}\right)=\operatorname{Cor}\left(\operatorname{Res}\left(\alpha_{i}\right) \cdot a_{j}\right)$. We find it helpful first to express $H^{*}(M)$ as a module over $H^{*}(G)$ so that Cor may be computed. (This task is made easier by our unambiguous description of each generator of $H^{*}(G)$.)

(1.11) The Steenrod Algebra $\mathscr{A}$. Since we rely frequently on the action of $\mathscr{A}$ on cohomology rings, we compute its action on the groups of order 32 . By virtue of the Cartan formula, it is sufficient to calculate its action on generators. The action of 
$\mathscr{A}$ commutes with inflation, restriction, and transfer, so for most of the generators there are no new techniques needed beyond those in (1.10).

For the Stiefel-Whitney classes $T_{i}$ we use the Wu formulae giving $S q^{i}\left(w_{j}(\rho)\right)$ in order to see that $S q^{i}(T)$ is always a multiple $w_{i}(\rho) \cdot T$ of the class $T$.

The $\mathrm{Wu}$ formulae (or the Adem relations) reduce our calculations to a determination of $w_{1}, w_{2}$, and $w_{4}$ (i.e., $S q^{1}(T), S q^{2}(T)$, and $S q^{4}(T)$ ). We can calculate $w_{1}(\rho)=w_{1}(\operatorname{det}(\rho))$ as the element $\operatorname{det}(\rho) \in \operatorname{Hom}\left(G, \mathbf{F}_{2}\right)=H^{1}(G)$. The remaining classes, when needed, were calculated by considering their restrictions to subgroups. Unfortunately, this left a few ambiguities which we did not feel inclined to resolve. (An asterisk in the tables of Section 3 indicates a coefficient of 0 or 1.)

(1.12) Other Computations. It should be possible to continue from the data given to compute other homological data, but we have not done so. Some results may be necessary, should one need to calculate the cohomology of still larger groups: we have seen it is necessary to know the restriction, inflation, and corestriction maps, as well as the action of group automorphisms.

In addition, one may wish to compute integral homology and cohomology (and, too, cohomology with coefficients). We have succeeded in applying the Bockstein spectral sequence in a few cases.

The author is willing to act as a clearinghouse for such data as they accumulate.

2. Conclusions Drawn from the Data. We use the groups of order 32 as a "database" on which to test old conjectures and formulate new ones. We incidentally verify our calculations by comparing our computed $H^{*}(G)$ to various known results.

In most cases, the reader can probably calculate the necessary data directly from the cohomology rings. We have provided the results of those calculations when space permitted. The commentary in Section 2 should provide enough guidance to understand the tables in Section 3.

(2.1) Generators and Relations. Each cohomology ring $H^{*}(G)$ may be written as a weighted polynomial ring modulo an ideal generated by finitely many homogeneous relations. We give in Section 3 such a presentation for each group of order 32. In column 1 we give the degrees of the generators, which will always be called $z$, $y, x, w, \ldots$ in order. In column 2 we give a minimal set of generators for the ideal of relations. From this perspective, the most complicated group is $\Gamma_{7} a_{3}$, which requires 9 generators for $H^{*}(G)$, bound by 27 relations.

The integers in column 1 are invariants of the group, that is, they do not depend on the choice of a minimal generating set for $H^{*}(G)$. It is not clear how to interpret these invariants in general, although the number of 1 's is equal to $d(G)$, the rank of $G / \Phi(G)$.

In column 3 we give the action of the Steenrod algebra by giving, for each generator $x \in H^{n}(G)$ with $n>1$, the images $S q^{i}(x)$ for $0 \leq i \leq n$.

Using these bits of data allows a few checks on our calculations. To verify the Cartan formula, we need to check that the ideal of relations is invariant under the total squaring operation (a ring homomorphism). This was checked with Macaulay. We also checked some Adem relations on the generators (e.g., $S q^{1} S q^{1}=0$ and $\left.S q^{1} S q^{2}=S q^{3}\right)$ and verified the instability axiom $\left(S q^{n} x=x^{2}\right)$ for elements of 
degree not a power of 2 . When convenient, we also verified that the ideal of relations restricted to zero on subgroups and that the Steenrod squares commuted with the restriction.

We observe that $H^{*}\left(G_{1}\right) \not H^{*}\left(G_{2}\right)$ for distinct groups $G_{i}$ of order 32 , except for the isomorphisms

$$
\begin{gathered}
H^{*}\left(\Gamma_{2} i\right) \cong H^{*}\left(\mathbb{Z}_{4} \times \mathbb{Z}_{8}\right), \\
H^{*}\left(\Gamma_{2} j_{2}\right) \cong H^{*}\left(\Gamma_{3} d_{1}\right) \cong H^{*}\left(\Gamma_{3} d_{2}\right),
\end{gathered}
$$

The first two of these are also isomorphisms of modules over the Steenrod algebra.

(2.2) Poincaré Series. The Poincaré series

$$
P_{G}(t)=\sum_{n \geq 0} t^{n} \cdot \operatorname{dim} H^{n}(G)
$$

is known to be a rational function whose only poles are $|G|$ th roots of unity. Thus, we may write $P_{G}(t)=N_{G}(t) \cdot \prod \phi_{k}^{n(k)}$, where $\phi_{k}$ is the $k$ th cyclotomic polynomial and $N_{G}$ is a polynomial over the integers coprime to all $\phi_{k}$. Column 4 gives the Poincaré series for each group as one of the twenty series in the accompanying table. Here $\psi_{n}$ is the following polynomial of degree $n$ :

$$
\begin{aligned}
& \psi_{3}(t)=1+t^{2}+t^{3} \\
& \psi_{4}(t)=1+t^{3}-t^{4}, \\
& \psi_{5}(t)=1+t^{2}+t^{5} \\
& \psi_{6}(t)=1+t+t^{2}+t^{5}+t^{6} .
\end{aligned}
$$

These polynomials each occur in only one group. At the other extreme, fully half of the groups have series $P_{G}=\phi_{1}^{-2}$ or $\phi_{1}^{-3}$.

It had previously been suggested that all zeros of $P_{G}$ were roots of unity as well, but we see now this is not the case. We do observe that they are all algebraic integers when $G$ has order at most 32 , although we have no proof that this is true for arbitrary groups.

The order $-n(1)$ of the pole at $t=1$ is the Krull dimension of $H^{*}(G)$, which is known [9] to be the rank of $G$; this agrees with the computed data. There is no interpretation known to the author of the other invariants-the locations and multiplicities of the zeros and poles. We might ask if there is a natural map from $\operatorname{Aut}(G)$ (or perhaps even $\operatorname{Out}(G)$ ) to the Galois group of $P_{G}$. The referee kindly pointed out that the Galois group of the polynomial $\psi_{n}$ listed above is the symmetric group $S_{n}$; in view of the known order of the automorphism groups of the groups $G$ in question (see [7]) it is thus clear that the image of Aut $(G)$ in the Galois group is comparatively small, at least in these cases.

We remark that one may view the "series" $\mathscr{K}_{G}(t)=\sum t^{n} \cdot H^{n}(G)$ of modules of $\operatorname{Aut}(G)$ (or Out $(G)$ ) as a finitely generated module over the (Aut $(G)$-invariant) 
Poincaré series occurring for groups of order 32

\begin{tabular}{|c|c|c|c|c|c|c|c|c|c|}
\hline Series & $n_{1}$ & $n_{2}$ & $n_{3}$ & $n_{4}$ & $n_{5}$ & $n_{6}$ & $n_{8}$ & $N_{G}$ & leading coeffs \\
\hline 1 & -1 & & & & & & & & \begin{tabular}{|lllllll}
1 & 1 & 1 & 1 & 1 & 1
\end{tabular} \\
\hline 2 & -1 & 0 & 1 & -1 & & & & & $\begin{array}{llllll}1 & 2 & 2 & 1 & 1 & 2\end{array}$ \\
\hline 3 & -2 & 0 & 0 & -1 & & & & & $\begin{array}{llllll}1 & 2 & 2 & 2 & 3 & 4\end{array}$ \\
\hline 4 & -2 & 0 & 0 & -1 & 0 & 0 & -1 & $\psi_{5}$ & 123445 \\
\hline 5 & -2 & & & & & & & & 1223456 \\
\hline 6 & -3 & 0 & 0 & -1 & 0 & 1 & & & 1235811 \\
\hline 7 & -3 & -1 & & & & & & & 1246912 \\
\hline 8 & -3 & -2 & 1 & & & & & & 12571215 \\
\hline 9 & -2 & 0 & 0 & -2 & 1 & & & & 134469 \\
\hline 10 & -3 & 0 & 0 & -2 & & & & $\psi_{4}$ & 1345811 \\
\hline 11 & -2 & 0 & 0 & -1 & 0 & 0 & -1 & $\psi_{6}$ & 1335667 \\
\hline 12 & -2 & 0 & 1 & -1 & & & & & 135679 \\
\hline 13 & -3 & 0 & 0 & -1 & & & & & 1355679 \\
\hline 14 & -3 & -1 & 0 & -1 & & & & $\psi_{3}$ & $\begin{array}{lllll}13 & 58 & 12 & 17\end{array}$ \\
\hline 15 & -3 & & & & & & & & 1336101521 \\
\hline 16 & -4 & -1 & & & & & & & 137132234 \\
\hline 17 & -2 & 0 & 1 & -1 & 1 & 0 & -1 & & 149152126 \\
\hline 18 & -3 & 0 & 1 & -1 & & & & & 149152231 \\
\hline 19 & -4 & & & & & & & & 1410203556 \\
\hline 20 & -5 & & & & & & & & 15153570126 \\
\hline
\end{tabular}

subring generated by the Stiefel-Whitney classes of the regular representation, so that

$$
\mathscr{H}_{G}(t)=\mathscr{N}_{G}(t) \cdot \prod \phi_{k}^{m(k)}
$$

where $\mathscr{N}_{G}$ is a "polynomial" with coefficients in the set of modules over $\operatorname{Aut}(G)$. We have not tried to compute $\mathscr{N}_{G}$ or the $m(k)$. For an example, see [5].

Observe that $P_{G}$ may be computed either from $E_{\infty}$ or $H^{*}(G)$. Miscalculation in the relations of the latter may produce higher syzygies that would affect $P_{G}$; we have checked that instead the proper series is obtained.

(2.3) Module Structure. It sometimes makes calculations easier if it is possible to find a pure polynomial subring $D=\mathrm{F}_{2}\left[u_{1}, \ldots, u_{n}\right] \subset H^{*}(G)$ over which $H^{*}(G)$ is a "nice" (e.g., finitely generated free) module (for example, it is easier to work with $16 \Gamma_{2} c_{1}$ in this way). In almost every case, we find such an algebra $D$ so that as $D$-modules,

$$
H^{*}(G)=M_{0} \oplus M_{1} \oplus \cdots \oplus M_{n}
$$

where each $M_{i}$ is a finitely-generated free module over $F_{2}\left[u_{1}, \ldots, u_{i}\right]$, but $u_{j} M_{i}=0$ when $j>i$. For groups of order 32, we either have $H^{*}(G)=M_{n}$ (that is, $H^{*}(G)$ is a Cohen-Macaulay ring) or $H^{*}(G)=M_{n-1} \oplus M_{n}$ (that is, depth $H^{*}(G)=$ $\operatorname{dim} H^{*}(G)-1$ ) except for the group $\Gamma_{7} a_{2}$ (which incidentally only has depth 1). 
For this odd group,

$$
H^{*}(G)=\mathbf{F}_{2}[z, x, s] \cdot(1, w, v, u, t) /(z \cdot u+x \cdot w) \oplus \mathbf{F}_{2}[s] \cdot y
$$

We have tried to suggest the module decompositions which we found in an economical way. In column 5 we give the degrees of the ring generators $u_{i}$. The module $M_{n}$ is generated by a finite number of classes $a_{i}$; in column 6 , we give the degrees of these classes. When $M_{n-1} \neq 0$, we give in column 7 the degree of the generator of the ideal $\operatorname{ann}_{D}\left(M_{n-1}\right)=\left(u_{n}\right)$, and the degrees of the generators of $M_{n-1}$.

The most complex group in this sense is $\Gamma_{5} a_{2}$, which can only be written as a free module over a pure polynomial subring if we use at least 120 module generators. The group $\Gamma_{4} d$ is also interesting: it allows such a description as a $D$-module where $D$ has a generator of odd degree.

Of course the integers in these columns are not invariants of $G$; but one can easily recoup the Poincaré series from them as a numerical check.

There has been some speculation regarding the depth of cohomology rings $H^{*}(G)$; unfortunately, the table shows there is not much variation in our data. We do check that the depth is at least the rank of the center [3]. We do also observe that $H^{*}(G)$ is not Cohen-Macaulay for any $G$ with a unique conjugacy class of (noncentral!) maximal elementary-abelian subgroups, nor for either $G$ with nonisomorphic maximal elementary subgroups $\left(\Gamma_{4} a_{1}\right.$ and $\left.\Gamma_{6} a_{1}\right)$. This supports the general consensus that depth is related to the placement of elementary-abelian subgroups in $G$.

(2.4) Ring Structure. The ideal structure of $H^{*}(G)$ (e.g., Spec $H^{*}(G)$ ) is known to be related to the elementary subgroups as well. In column 8 we give generators of the nilpotent radical $\sqrt{0} \subset H^{*}(G)$, and in column 9 we write this as antersection of minimal prime ideals. These are precisely the minimal primes which are invariant under the action of the Steenrod algebra. Quillen [9] showed that we may associate each such prime to a conjugacy class of maximal elementary subgroups $E$ and that $H^{*}(G) / P$ is roughly isomorphic to the ring of invariants $H^{*}(E)^{N(E)}$. Among groups of order 32 , all such rings of invariants will be pure polynomial rings except for those in $\Gamma_{4} a_{1}, \Gamma_{7} a_{1}$, and $\Gamma_{7} a_{2}$. The apparent anomaly in $\Gamma_{6} a_{1}$ results from the more precise statement that $H^{*}(G)$ is isogenous to

$$
\lim _{\leftarrow} H^{*}(E)^{N_{G}(E)} \text {. }
$$

We have not yet calculated for all $G$ the square roots necessary to obtain (**) from $H^{*}(G) / \sqrt{0}$. We do observe that in some cases (e.g., $\Gamma_{2} c_{1}$ ) the algebraic adjunction of square roots adds nilpotent elements, since $H^{*}(G) / \sqrt{0}$ is not integrally closed; thus $(* *)$ is obtained from $H^{*}(G) / \sqrt{0}$ only by the adjunction of square roots within $(* *)$.

(2.5) Detection. We have seen how useful it is in the calculation of $H^{*}(G)$ to have a family of subgroups $H<G$ which detect $H^{*}(G)$. For some groups (called essential groups) no such family exists; we let $\operatorname{Ess}(G) \subseteq H^{*}(G)$ be the ideal of essential elements (restricting to zero on all subgroups). This is $\bigcap(L): L \in H^{1}(G)$. 
In column 10, we give for essential groups a minimal set of generators of $\operatorname{Ess}(G)$. For nonessential groups, we give a minimal list of $L \in H^{1}(G)$ for which $\bigcap(L)=(0)$. (Thus, the maximal subgroups $\operatorname{Ker}(L)$ detect.) This list is not in general unique; even its cardinality may vary.

Frequently, the subgroups $\operatorname{Ker}(L)$ are not essential either, and so the cohomology is further detected by proper subgroups of it. We give in column 11 the isomorphism types of subgroups in a detecting set which are themselves essential. (The set contains no pairs conjugate to or contained in each other, but it is possible that the set is not minimal.) We remark that these subgroups are not even unique up to conjugacy (e.g., for $G=\Gamma_{2} c_{1}$ ). We write $Q_{n}$ for the quaternion group of order $n, Q D$ for the quasi-dihedral groups, and $A . B$ for a split (noncommuting) product.

The ideal case is perhaps one in which elementary-abelian subgroups detect. This happens if and only if $\sqrt{0}=0$, and so we observe from the table only a few such groups:

$$
\mathbb{Z}_{2}^{5}, \mathbb{Z}_{2}^{3} \times D_{8}, \mathbb{Z}_{2} \times D_{16}, \Gamma_{5} a_{1}=D_{8} \circ D_{8}, \Gamma_{4} a_{1}, \Gamma_{4} a_{2}, \Gamma_{4} b_{1}, D_{32}
$$

As with other phenomena, there is expected to be a link between the existence of essential elements in $H^{*}(G)$ and the placement of the elementary subgroups and their centralizers. For example, it is conjectured that when all involutions are central, $G$ is essential. This is verified when $|G|=32$. In other direction, it is felt that if $G$ is essential, there should conversely be few involutions, with large centralizers - e.g., they should all commute with each other (form a subgroup). Indeed, this property is held by the essential groups

$$
\Gamma_{2} d, \Gamma_{2} f, \Gamma_{2} k, \Gamma_{3} f, \Gamma_{4} d, \Gamma_{7} a_{3}
$$

(and the nonessential groups $\Gamma_{2} c_{1}, \Gamma_{2} j$, and $\Gamma_{4} b_{2}$ ). The lone surprise is the group $\Gamma_{6} a_{2}$ which is essential but whose involutions do not form a subgroup. It is not now clear what would be a reasonable conjecture in this direction.

(2.6) Further Remarks on the Table. In column 12 we indicate the set of maximal elementary-abelian subgroups. The data are taken from [7]. The entries have one of the following forms:

$n \mathrm{I}$ : all involutions are central; $\operatorname{rank}(G)=n$.

$n$ II: the involutions form a noncentral subgroup $E$ whose rank is $n$.

$n$ III: all maximal elementary subgroups $E$ are conjugate, not normal, and of rank $n$.

$n \mathrm{IV} m$ : there exist $m$ maximal elementary subgroups, all normal and of rank $n$.

$n \mathrm{~V} m_{1} m_{2} \ldots$ : all maximal elementary subgroups have rank $n$ and lie in conjugacy classes of cardinalities $m_{1}, m_{2}, \ldots$

$n$ VI: there exist at least two nonisomorphic maximal elementary subgroups; $G$ has rank $n$. 
For every maximal elementary subgroup $E$ in a group $G$ of class II or IV, $[E: E \cap Z(G)]=2$, and $[G: C(E)]=2$ except when $G=\Gamma_{4} d, \Gamma_{7} a_{1}$, or $\Gamma_{7} a_{2}$, in which case $E$ is self-centralizing and $G / E$ is cyclic. In class III, $E$ is self-centralizing and $[N(E): E]=2$. Class VI contains only two groups. $\Gamma_{4} a_{1}$ has two maximal elementary subgroups, both normal: $E_{1}$ of rank 4 and $E_{2}$ of rank 3, meeting in the center (of rank 2). We have $G / C\left(E_{1}\right) \cong \mathbb{Z}_{2}$ acting with a fixed subspace of rank 2 in $\mathbf{Z}_{2}^{4}$, and $G / C\left(E_{2}\right) \cong \mathbf{Z}_{2}^{2}$ acting faithfully on $\mathbf{Z}_{2}^{3}$. The other group in class VI is $\Gamma_{6} a_{1}$, which has two conjugacy classes of maximal elementary subgroups, $E_{1}$ of rank 2 and $E_{2}$ of rank 3 . In each case $[G: N(E)]=2,[N(E): C(E)]=2$, and $\left[E: E \cap E^{g}\right]=2$.

In column 13 we give references to the specific groups in the body of the text, and in column 14 we refer to previous published treatments of which we are aware of the given groups. (Direct products can of course be readily handled when the factors have been discussed in the literature.)

(2.7) Further Directions. There are several more areas of current research for which we hope the attached tables will be of use.

There has been considerable effort expended in describing a stable decomposition of $B G=\bigvee X_{i}$ with indecomposable spectra $X_{i}$. As a first step, we would like to calculate a decomposition of $H^{*}(G)$ into indecomposable summands over $\mathscr{A}$.

It would be most pleasant if a complete set of generators of cohomology rings were easily available. A frequent choice is the set of Stiefel-Whitney classes of representations. We have not calculated all the classes for all the representations, but it may turn out that among the "lower" classes $\left(w_{k}(\rho)\right.$ where $\left.k<\operatorname{deg}(\rho)\right)$ we will encounter more generators for $H^{*}(G)$.

Certainly, we know that we will not recover all of $H^{*}(G)$ in this way. A more promising possibility arises from the modular representations $\rho: G \rightarrow G L\left(n, \mathbf{F}_{2}\right)$. Do the images of the various maps $\rho^{*}$ generate $H^{*}(G)$ ? Perhaps our expansionrestriction technique is best embodied by two conjectures along these lines: let $U_{n}$ be the unipotent (= Sylow) subgroup of $G L\left(n, \mathbf{F}_{2}\right)$ and ask

CONJECTURE 1. Is $H^{*}\left(U_{n}\right)$ detected by its elementary-abelian subgroups?

CONJECTURE 2. For every 2-group $G$, is there a (modular) representation $\rho$ : $G \rightarrow U_{n}$ such that $\rho^{*}: H^{*}\left(U_{n}\right) \rightarrow H^{*}(G)$ is onto?

(Such a $\rho$ is necessarily injective.)

3. Appended Data. This last section consists only of the appended tables. For each of the 51 groups of order 32 we give various pieces of data in columns. The column headings are cryptic reminders of the descriptions given in Section 2. 


\section{Group Data}

\begin{tabular}{|c|c|c|}
\hline $\begin{array}{l}\text { 1. Group } \\
\text { \& Degs. gens. }\end{array}$ & 2. Ring Relations & $\begin{array}{c}\text { 3. Total Steenrod } \\
\text { Squares }\end{array}$ \\
\hline $\begin{array}{l}(5) \\
12\end{array}$ & $z^{2}$ & $y, 0, y^{2}$ \\
\hline $\begin{array}{l}(41) \\
112\end{array}$ & $y^{2}$ & $x, 0, x^{2}$ \\
\hline $\begin{array}{l}(32) \\
1122\end{array}$ & $\begin{array}{l}z^{2} \\
y^{2}\end{array}$ & $\begin{array}{l}x, 0, x^{2} \\
w, 0, w^{2}\end{array}$ \\
\hline $\begin{array}{l}311) \\
1112 \\
\end{array}$ & $x^{2}$ & $w, 0, w^{2}$ \\
\hline $\begin{array}{l}(221) \\
11122\end{array}$ & $\begin{array}{l}y^{2} \\
x^{2}\end{array}$ & $\begin{array}{l}w, 0, w^{2} \\
v, 0, v^{2}\end{array}$ \\
\hline $\begin{array}{l}(2111) \\
11112 \\
\end{array}$ & $w^{2}$ & $v, 0, v^{2}$ \\
\hline \begin{tabular}{|l|}
$(11111)$ \\
11111 \\
\end{tabular} & no relations & \\
\hline $\begin{array}{l}\Gamma_{2} a_{1} \\
11112 \\
\end{array}$ & $z y+y^{2}$ & $v, z v, v^{2}$ \\
\hline $\begin{array}{l}\Gamma_{2} a_{2} \\
11114\end{array}$ & $\begin{array}{l}x^{2}+x w+w^{2} \\
x^{3}\end{array}$ & $v, 0,0,0, v^{2}$ \\
\hline \begin{tabular}{|l|}
$\Gamma_{2} b$ \\
11114
\end{tabular} & $\begin{array}{l}y x+w^{2} \\
y^{2} x+y x^{2}\end{array}$ & $v, 0, y^{2} v+x^{2} v+w^{2} v, 0, v^{2}$ \\
\hline \begin{tabular}{|l|}
$\Gamma_{2} c_{1}$ \\
111222
\end{tabular} & $\begin{array}{l}y x \\
x^{2} \\
x w \\
y^{2} v+w^{2}\end{array}$ & $\begin{array}{l}w, y w+y v+x u, w^{2} \\
v, 0, v^{2} \\
u, y u+x u, u^{2}\end{array}$ \\
\hline $\begin{array}{l}\Gamma_{2} c_{2} \\
11122\end{array}$ & $\begin{array}{l}y^{2} \\
y x+x^{2}\end{array}$ & $\begin{array}{l}w, 0, w^{2} \\
v, y v, v^{2}\end{array}$ \\
\hline $\begin{array}{l}\Gamma_{2} d \\
11134\end{array}$ & $\begin{array}{l}y^{2} \\
y x^{2} \\
y w \\
w^{2}\end{array}$ & $\begin{array}{l}w, 0, x^{2} w+y v, w^{2} \\
v, 0, y x v+x^{2} v, 0, v^{2}\end{array}$ \\
\hline $\begin{array}{l}\Gamma_{2} e_{1} \\
11122\end{array}$ & $\begin{array}{l}z^{2} \\
y^{2}+y x\end{array}$ & $\begin{array}{l}w, 0, w^{2} \\
v, x v, v^{2}\end{array}$ \\
\hline \begin{tabular}{|l|}
$\Gamma_{2} e_{2}$ \\
11124
\end{tabular} & $\begin{array}{l}z^{2} \\
y^{2}+y x+x^{2} \\
y^{3}\end{array}$ & $\begin{array}{l}w, 0, w^{2} \\
v, 0,0,0, v^{2}\end{array}$ \\
\hline
\end{tabular}




\begin{tabular}{|c|c|c|}
\hline $\begin{array}{l}\text { 1. Group } \\
\text { \& Degs. gens. }\end{array}$ & 2. Ring Relations & $\begin{array}{l}\text { 3. Total Steenrod } \\
\text { Squares }\end{array}$ \\
\hline $\begin{array}{l}\Gamma_{2} f \\
111234\end{array}$ & $\begin{array}{l}y^{2} \\
z y+x^{2} \\
z^{2} y \\
y v \\
z^{4} w+v^{2}\end{array}$ & $\begin{array}{l}w, 0, w^{2} \\
v, z^{2} w+z y w, z^{2} v+y u, v^{2} \\
u, 0, z^{2} u+z y u, 0, u^{2}\end{array}$ \\
\hline $\begin{array}{l}\Gamma_{2} g \\
1114\end{array}$ & $\begin{array}{l}x^{2} \\
z^{2} y+z y^{2}\end{array}$ & $w, 0, z^{2} w+z y w+y^{2} w, 0, w^{2}$ \\
\hline $\begin{array}{l}\Gamma_{2} h \\
1122222\end{array}$ & $\begin{array}{l}z^{2} \\
y^{2} \\
z y, \\
u^{2} \\
t^{2} \\
u t \\
z u, \\
y t \\
y u+z t\end{array}$ & $\begin{array}{l}x, 0, x^{2} \\
w, 0, w^{2} \\
v, z v+y v, v^{2} \\
u, y x+z v, u^{2} \\
t, z w+y v, t^{2}\end{array}$ \\
\hline $\begin{array}{l}\Gamma_{2} i \\
1122\end{array}$ & $\begin{array}{l}z^{2} \\
y^{2}\end{array}$ & $\begin{array}{l}x, 0, x^{2} \\
w, 0, w^{2}\end{array}$ \\
\hline $\begin{array}{l}\Gamma_{2} j_{1} \\
11222\end{array}$ & $\begin{array}{l}z y \\
y^{2} \\
y x \\
x^{2}\end{array}$ & $\begin{array}{l}x, z x+y w, x^{2} \\
w, z w+y w, w^{2} \\
v, 0, v^{2}\end{array}$ \\
\hline $\begin{array}{l}\Gamma_{2 j} j_{2} \\
1122\end{array}$ & $\begin{array}{l}z^{2} \\
z y+y^{2}\end{array}$ & $\begin{array}{l}x, 0, x^{2} \\
w, z w, w^{2}\end{array}$ \\
\hline $\begin{array}{l}\Gamma_{2} k \\
1134\end{array}$ & $\begin{array}{l}z^{2} \\
z y^{2} \\
z x \\
x^{2} \\
\end{array}$ & $\begin{array}{l}x, 0, y^{2} x+z w, x^{2} \\
w, 0, z y w+y^{2} w, 0, w^{2}\end{array}$ \\
\hline $\begin{array}{l}\Gamma_{3} a_{1} \\
1112 \\
\end{array}$ & $y x+x^{2}$ & $w, y w, w^{2}$ \\
\hline $\begin{array}{l}\Gamma_{3} a_{2} \\
11134\end{array}$ & $\begin{array}{l}y x+x^{2} \\
y^{2} x \\
y^{2} v+y x v+w^{2} \\
x w\end{array}$ & $\begin{array}{l}w, 0, y^{2} w+y v+x v, w^{2} \\
v, 0, y^{2} v+y x v, 0, v^{2}\end{array}$ \\
\hline $\begin{array}{l}\Gamma_{3} a_{3} \\
1114\end{array}$ & $\begin{array}{l}y x+x^{2} \\
y^{3}\end{array}$ & $w, 0,0,0, w^{2}$ \\
\hline $\begin{array}{l}\Gamma_{3} b \\
1114\end{array}$ & $\begin{array}{l}z y+y^{2}+y x \\
z^{3}+z^{2} x\end{array}$ & $w, 0, x^{2} w, 0, w^{2}$ \\
\hline
\end{tabular}




\begin{tabular}{|c|c|c|}
\hline $\begin{array}{l}\text { 1. Group } \\
\text { \& Degs. gens. }\end{array}$ & 2. Ring Relations & $\begin{array}{c}\text { 3. Total Steenrod } \\
\text { Squares }\end{array}$ \\
\hline $\begin{array}{l}\Gamma_{3} c_{1} \\
11222\end{array}$ & $\begin{array}{l}z y \\
y^{2} \\
y x \\
z^{2} w+x^{2}\end{array}$ & $\begin{array}{l}x, y x+y w, x^{2} \\
w, 0, w^{2} \\
v, z v+y v, v^{2}\end{array}$ \\
\hline $\begin{array}{l}\Gamma_{3} c_{2} \\
112234\end{array}$ & $\begin{array}{l}z y \\
z^{2} \\
y^{3} \\
z w \\
y v \\
y^{2} x+w^{2} \\
v^{2} \\
w v \\
y^{2} w+z v\end{array}$ & $\begin{array}{l}x, 0, x^{2} \\
w, y x+y w, w^{2} \\
v, 0,0, v^{2} \\
u, 0,0,0, u^{2}\end{array}$ \\
\hline $\begin{array}{l}\Gamma_{3} d_{1} \\
1122\end{array}$ & $\begin{array}{l}z^{2} \\
z y+y^{2}\end{array}$ & $\begin{array}{l}x, 0, x^{2} \\
w, z w, w^{2}\end{array}$ \\
\hline $\begin{array}{l}\Gamma_{3} d_{2} \\
1122\end{array}$ & $\begin{array}{l}z^{2} \\
z y+y^{2}\end{array}$ & $\begin{array}{l}x, 0, x^{2} \\
w, y x+z w, w^{2}\end{array}$ \\
\hline $\begin{array}{l}\Gamma_{3} e \\
112234\end{array}$ & $\begin{array}{l}z y \\
y^{2} \\
y x \\
x^{2} \\
z w \\
z v \\
x w+y v \\
v^{2} \\
x v\end{array}$ & $\begin{array}{l}x, z x+y x, x^{2} \\
w, 0, w^{2} \\
v, 0, w v+y u, v^{2} \\
u, 0, z^{2} u+w u, 0, u^{2}\end{array}$ \\
\hline $\begin{array}{l}\Gamma_{3} f \\
11234\end{array}$ & $\begin{array}{l}z^{2} \\
z y+y^{2} \\
z x \\
z w \\
w^{2}\end{array}$ & $\begin{array}{l}x, 0, x^{2} \\
w, 0, x w+z v, w^{2} \\
v, 0, x v, 0, v^{2}\end{array}$ \\
\hline $\begin{array}{l}\Gamma_{4} a_{1} \\
111222\end{array}$ & $\begin{array}{l}z y, \\
y x \\
y u, \\
x^{2} w+z^{2} v+z x u+u^{2}\end{array}$ & $\begin{array}{l}w, z w+y w, w^{2} \\
v, y v+x v, v^{2} \\
u, x w+z v+z u+x u, u^{2}\end{array}$ \\
\hline $\begin{array}{l}\Gamma_{4} a_{2} \\
11122\end{array}$ & $\begin{array}{l}z^{2}+z x \\
y^{2}+y x\end{array}$ & $\begin{array}{l}w, x w, w^{2} \\
v, x v, v^{2}\end{array}$ \\
\hline
\end{tabular}




\begin{tabular}{|c|c|c|}
\hline \begin{tabular}{|l} 
1. Group \\
\& Degs. gens.
\end{tabular} & 2. Ring Relations & $\begin{array}{c}\text { 3. Total Steenrod } \\
\text { Squares }\end{array}$ \\
\hline $\begin{array}{l}\Gamma_{4} a_{3} \\
11124\end{array}$ & $\begin{array}{l}z^{2}+z x+x^{2} \\
z y+y^{2} \\
z^{3}\end{array}$ & $\begin{array}{l}w, z w, w^{2} \\
v, 0,0,0, v^{2}\end{array}$ \\
\hline $\begin{array}{l}\Gamma_{4} b_{1} \\
11122\end{array}$ & $\begin{array}{l}y x+x^{2} \\
z y\end{array}$ & $\begin{array}{l}w, y w, w^{2} \\
v, z v+y v, v^{2}\end{array}$ \\
\hline $\begin{array}{l}\Gamma_{4} b_{2} \\
111234\end{array}$ & $\begin{array}{l}y^{2}+y x+x^{2} \\
y^{3} \\
z y \\
y v \\
z^{2} u+v^{2}\end{array}$ & $\begin{array}{l}w, z w+y w, w^{2} \\
v, y^{2} w+z v, z u, v^{2} \\
u, 0,0,0, u^{2}\end{array}$ \\
\hline $\begin{array}{l}\Gamma_{4} c_{1} \\
1112334\end{array}$ & $\begin{array}{l}z^{2}+y x \\
z y \\
y x^{2} \\
y v \\
z v+x u \\
z u \\
x^{4} w+x^{3} u+y x t+v^{2} \\
y^{2} t+u^{2} \\
z x^{3} w+v u\end{array}$ & $\begin{array}{l}w, z w+y w, w^{2} \\
v, y x w+x^{2} w+x u, x^{2} v+z t, v^{2} \\
u, z x w+x u, z x^{2} w+y^{2} u+y t \\
\quad u^{2} \\
t, 0, y^{2} t+y x t+x^{2} t, 0, t^{2}\end{array}$ \\
\hline $\begin{array}{l}\Gamma_{4} c_{2} \\
111234\end{array}$ & $\begin{array}{l}z y+z x+x^{2} \\
z y+y^{2} \\
z^{2} y \\
y v \\
z^{2} u+z y u+v^{2}\end{array}$ & $\begin{array}{l}w, z w+y w, w^{2} \\
v, z y w, z^{2} v+z u+y u, v^{2} \\
u, 0, z^{2} u+z x u+x^{2} u, 0, u^{2}\end{array}$ \\
\hline $\begin{array}{l}\Gamma_{4} c_{3} \\
1113344\end{array}$ & $\begin{array}{l}y^{2}+z x+x^{2} \\
z^{2}+z y+y^{2} \\
z^{3}+z^{2} y \\
z^{3} \\
z w+z v+y v \\
y w+z v \\
z^{2} v+z y v \\
z^{2} u+z y u+z^{2} t+w^{2} \\
z y u+z^{2} t+z y t+v^{2} \\
z^{2} u+z y t+w v\end{array}$ & $\begin{array}{l}w, y w+z v, y u+z t+y^{2} w, w^{2} \\
v, z v+y v, z u+y u+y t, v^{2} \\
u, 0,0,0, u^{2} \\
t, 0, y^{2} t, 0, t^{2}\end{array}$ \\
\hline
\end{tabular}




\begin{tabular}{|c|c|c|}
\hline $\begin{array}{l}\text { 1. Group } \\
\& \text { Degs. gens. }\end{array}$ & 2. Ring Relations & $\begin{array}{l}\text { 3. Total Steenrod } \\
\text { Squares }\end{array}$ \\
\hline $\begin{array}{l}\Gamma_{4} d \\
11133344\end{array}$ & $\begin{array}{l}y^{2}+z x \\
z y+z x+x^{2} \\
z^{2} x \\
z^{2} y \\
z w+y u \\
y w+x u \\
z w+x w+y v \\
z w+x v \\
z^{2} w \\
z y w+y x w \\
z y t+z x t+z x s+w^{2} \\
z^{2} t+z^{2} s+z y s+z x s+v^{2} \\
z x t+z^{2} s+u^{2} \\
z x t+z y s+y x s+w v \\
y x t+z y s+w u\end{array}$ & $\begin{array}{l}w, z w, z y w+z x w+x t+y s, w^{2} \\
v, z w, z^{2} v+z t+z s+x s, v^{2} \\
u, z w, z^{2} u+y t+z s, u^{2} \\
t, 0, z^{2} t+z y t, z^{2} y t+z y^{2} t, t^{2} \\
s, 0, z^{2} s+z x s, z^{2} x s+z x^{2} s, s^{2}\end{array}$ \\
\hline $\begin{array}{l}\Gamma_{5} a_{1} \\
11114\end{array}$ & $\begin{array}{l}y x+z w \\
y^{2} x+y x^{2}+z^{2} w+z w^{2}\end{array}$ & $\begin{array}{l}v, 0 \\
z^{2} v+z y v+y^{2} v+y w v+w^{2} v \\
z^{2} y v+z y^{2} v+y^{2} w v+y w^{2} v \\
v^{2}\end{array}$ \\
\hline $\begin{array}{l}\Gamma_{5} a_{2} \\
11118\end{array}$ & $\begin{array}{l}z y+x^{2}+x w+w^{2} \\
z^{2} y+z y^{2}+x^{2} w+x w^{2} \\
z^{4} y+z y^{4}+x^{4} w+x w^{4}\end{array}$ & $\begin{array}{l}v, 0,0,0, z w^{3} t+y w^{3} t+x w^{3} t+ \\
\quad z^{4} t+y^{4} t+x^{4} t+w^{4} t \\
z^{2} w^{3} t+y^{2} w^{3} t+x^{2} w^{3} t+ \\
z w^{4} t+y w^{4} t+x w^{4} t \\
x w^{5} t+y w^{5} t+z w^{5} t+ \\
x^{2} w^{4} t+y^{2} w^{4} t+z^{2} w^{4} t \\
z^{2} w^{5} t+y^{2} w^{5} t+x^{2} w^{5} t+ \\
z w^{6} t+y w^{6} t+x w^{6} t, v^{2}\end{array}$ \\
\hline \begin{tabular}{|l|}
$\Gamma_{6} a_{1}$ \\
11134
\end{tabular} & $\begin{array}{l}y x^{2} \\
z y \\
y w \\
z^{2} x w+z x^{2} w+z^{2} v+w^{2}\end{array}$ & $\begin{array}{c}w, 0, z^{2} w+z x w+x^{2} w+z v, w^{2} \\
v, 0, z^{2} v+z x v+y x v+x^{2} v \\
z^{2} x v+y^{2} x v+z x^{2} v, v^{2}\end{array}$ \\
\hline $\begin{array}{l}\Gamma_{6} a_{2} \\
111558\end{array}$ & $\begin{array}{l}y x \\
y^{3}+z^{2} x+x^{3} \\
z^{2} y^{3} \\
x w \\
y v \\
y^{2} w+z^{2} v+x^{2} v \\
w v \\
z^{8} y^{2}+z^{4} y w+y^{2} u+w^{2} \\
x^{2} u+v^{2}\end{array}$ & $\begin{array}{l}w, z^{4} y^{2}+y w, 0,0, z^{4} w+y u, w^{2} \\
v, x v, z^{2} v, z^{2} x v, x u, v^{2} \\
u, 0,0,0, z^{4} u+(*) z y^{3} u, 0,0,0 \\
\quad u^{2}\end{array}$ \\
\hline
\end{tabular}




\begin{tabular}{|c|c|c|}
\hline $\begin{array}{l}\text { 1. Group } \\
\text { \& Degs. gens. }\end{array}$ & 2. Ring Relations & $\begin{array}{l}\text { 3. Total Steenrod } \\
\text { Squares }\end{array}$ \\
\hline \begin{tabular}{|l|}
$\Gamma_{7} a_{1}$ \\
11222334
\end{tabular} & $\begin{array}{l}z y, \\
y^{2}, \\
y v, \\
y u, \\
y t, \\
z^{3}+z x+y x+z w, \\
z x+y x+z v, \\
z u, \\
z^{2} t+x u+v u+x t+w t, \\
x u+x t+v t, \\
x^{2} w+x w v+u t, \\
z^{2} x+x w+v^{2}, \\
z^{4} x+z^{2} x^{2}+x^{2} w+x w^{2}+u^{2}, \\
z^{2} x^{2}+x^{2} w+z x t+z^{2} s+t^{2}\end{array}$ & $\begin{array}{l}x, z x, x^{2} \\
w, z x, w^{2} \\
v, z x+y x+u, v^{2} \\
u, 0 \\
\quad z^{2} u+z^{2} t+w u+x t+w t+y s \\
\quad u^{2} \\
t, z^{2} x+x w \\
\quad z x^{2}+y x^{2}+z^{2} t+x t+z s, t^{2} \\
s, y s, x s+w s+v s \\
\quad u s+z x s+y w s, s^{2}\end{array}$ \\
\hline $\begin{array}{l}\Gamma_{7} a_{2} \\
11223344\end{array}$ & $\begin{array}{l}z y \\
y^{2} \\
y x \\
y w \\
y v \\
y u \\
y t \\
x w+z u \\
z^{2} w+w^{2} \\
w v+z t \\
z x w+w u \\
z^{2} t+w t \\
x^{3}+z x v+z^{2} s+v^{2} \\
v u+x t \\
x^{2} u+z x t+z w s+v t \\
x^{3} w+z^{2} x t+z^{2} w s+t^{2} \\
z x t+u t \\
x^{2} w+u^{2}\end{array}$ & $\begin{array}{l}x, z x+y x, x^{2} \\
w, 0, w^{2} \\
v, x^{2}, z^{2} v+x^{2} z+x v+z s, v^{2} \\
u, 0, z x w+x u, u^{2} \\
\quad t, x u+z t, z^{2} t+x t+w s \\
\quad z x^{2} w+z^{3} t+x^{2} u, t^{2} \\
s, 0, z^{2} s+x s, z x s, s^{2}\end{array}$ \\
\hline
\end{tabular}




\begin{tabular}{|c|c|c|}
\hline $\begin{array}{l}\text { 1. Group } \\
\text { \& Degs. gens. }\end{array}$ & 2. Ring Relations & $\begin{array}{c}\text { 3. Total Steenrod } \\
\text { Squares }\end{array}$ \\
\hline \begin{tabular}{|l}
$\Gamma_{7} a_{3}$ \\
112235568
\end{tabular} & $\begin{array}{l}x^{2} w+v^{2} \\
z y \\
y^{2} \\
z^{3}+y x \\
y w \\
z^{2} w+y v \\
z^{2} x+x w+z v \\
w^{2}+z^{2} w \\
y x^{2} \\
w v \\
z x v+y x v+z u \\
y u \\
y t \\
y s \\
w u \\
z^{2} t+w t+z s \\
x^{2} v+z^{2} u+x u+w t+z s \\
w s \\
x^{3} w+z^{2} s+v u \\
z x u+z^{2} s+v u+v t+x s \\
z v u+x w t+v s \\
x v u+z v s+u^{2} \\
x^{2} s+z v s+u t \\
x^{5}+z x^{2} t+z^{2} r+t^{2} \\
v^{2} t+u s \\
x^{3} u+z x^{2} s+x v s+y x r+ \\
z w r+u s+t s \\
w t^{2}+s^{2} \\
z w \\
z\end{array}$ & $\begin{array}{l}x, z x+y x, x^{2} \\
w, 0, w^{2} \\
v,(*) z^{2} w, u, v^{2} \\
u, z^{2} x^{2}+z u, 0,0 \\
\quad z x^{3} w+y r+x^{2} u, u^{2} \\
t, z^{2} x^{2}+x^{3}+z t+(*) z^{2} w x, 0,0 \\
\quad z x^{3} w+x^{3} v+x^{2} u+x^{2} t+z r \\
\quad t^{2} \\
s, z x^{2} w+x^{2} v, x^{3} w+z w t, 0 \\
\quad z x w t+x^{2} s+z^{2} r+w r, x^{4} v \\
\quad s^{2} \\
r, 0,0,0, z^{2} x r+x^{2} r+(*) z^{2} w r \\
0,0,0, r^{2}\end{array}$ \\
\hline $\begin{array}{l}\Gamma_{8} a_{1} \\
112\end{array}$ & $z y+y^{2}$ & $x, z x, x^{2}$ \\
\hline $\begin{array}{l}\Gamma_{8} a_{2} \\
1134\end{array}$ & $\begin{array}{l}z y+y^{2} \\
y^{3} \\
y x \\
z^{2} w+y^{2} w+x^{2}\end{array}$ & $\begin{array}{l}x, 0, z^{2} x+z w+y w, x^{2} \\
w, 0, z^{2} w+z y w, 0, w^{2}\end{array}$ \\
\hline $\begin{array}{l}\Gamma_{8} a_{3} \\
114\end{array}$ & $\begin{array}{l}z y+y^{2} \\
z^{3}\end{array}$ & $x, 0,0,0, x^{2}$ \\
\hline
\end{tabular}




\begin{tabular}{|l|l|l|l|l|}
\hline 1. Group & $\begin{array}{c}4 . \text { Poinc. } \\
\text { Series }\end{array}$ & $\begin{array}{c}\text { 5. Polynom } \\
\text { subring }\end{array}$ & $\begin{array}{l}\text { 6. Module } \\
\text { gens. }\end{array}$ & $\begin{array}{l}\text { 7. 2nd Mod. } \\
\text { gens. }\end{array}$ \\
\hline$(5)$ & 1 & 2 & 11 & - \\
\hline$(41)$ & 5 & 12 & 11 & - \\
\hline$(32)$ & 5 & 22 & 121 & - \\
\hline$(311)$ & 15 & 112 & 11 & - \\
\hline$(221)$ & 15 & 122 & 121 & - \\
\hline$(2111)$ & 19 & 1112 & 11 & - \\
\hline$(11111)$ & 20 & 1111 & 1 & - \\
\hline$\Gamma_{2} a_{1}$ & 19 & 1112 & 11 & - \\
\hline$\Gamma_{2} a_{2}$ & 18 & 114 & 1221 & - \\
\hline$\Gamma_{2} b$ & 18 & 124 & 13431 & - \\
\hline$\Gamma_{2} c_{1}$ & 16 & 1122 & 1001 & $(1) 01$ \\
\hline$\Gamma_{2} c_{2}$ & 15 & 122 & 121 & - \\
\hline$\Gamma_{2} d$ & 13 & 124 & 11011 & $(2) 011$ \\
\hline$\Gamma_{2} e_{1}$ & 15 & 122 & 121 & - \\
\hline$\Gamma_{2} e_{2}$ & 12 & 24 & 13431 & - \\
\hline$\Gamma_{2} f$ & 13 & 224 & 121121 & $(2) 0121$ \\
\hline$\Gamma_{2} g$ & 12 & 24 & 13431 & - \\
\hline$\Gamma_{2} h$ & 8 & 222 & 1221 & - \\
\hline$\Gamma_{2} i$ & 5 & 22 & 121 & - \\
\hline$\Gamma_{2} j_{1}$ & 7 & 122 & 101 & $(1) 01$ \\
\hline$\Gamma_{2} j_{2}$ & 5 & 22 & 121 & - \\
\hline$\Gamma_{2} k$ & 3 & 24 & 11011 & $(2) 011$ \\
\hline$\Gamma_{3} a_{1}$ & 15 & 112 & 11 & - \\
\hline$\Gamma_{3} a_{2}$ & 13 & 124 & 11011 & $(2) 011$ \\
\hline$\Gamma_{3} a_{3}$ & 12 & 14 & 1221 & - \\
\hline$\Gamma_{3} b$ & 12 & 14 & 1221 & - \\
\hline$\Gamma_{3} c_{1}$ & 7 & 122 & 101 & $(1) 01$ \\
\hline$\Gamma_{3} c_{2}$ & 5 & 24 & 12221 & - \\
\hline$\Gamma_{3} d_{1}$ & 5 & 22 & 121 & - \\
\hline$\Gamma_{3} d_{2}$ & 5 & 22 & 121 & - \\
\hline$\Gamma_{3} e$ & 5 & 24 & 12221 & - \\
\hline$\Gamma_{3} f$ & 3 & 14 & 11011 & $(1) 011$ \\
\hline & & & & \\
\hline
\end{tabular}




\begin{tabular}{|l|l|l|l|l|}
\hline 1. Group & $\begin{array}{c}4 . \text { Poinc. } \\
\text { Series }\end{array}$ & $\begin{array}{l}\text { 5. Polynom } \\
\text { subring }\end{array}$ & $\begin{array}{c}\text { 6. Module } \\
\text { gens. }\end{array}$ & $\begin{array}{l}\text { 7. 2nd Mod. } \\
\text { gens. }\end{array}$ \\
\hline$\Gamma_{4} a_{1}$ & 16 & 1122 & 101 & $(1) 01$ \\
\hline$\Gamma_{4} a_{2}$ & 15 & 122 & 121 & - \\
\hline$\Gamma_{4} a_{3}$ & 12 & 24 & 13431 & - \\
\hline$\Gamma_{4} b_{1}$ & 15 & 122 & 121 & - \\
\hline$\Gamma_{4} b_{2}$ & 13 & 124 & 11011 & $(1) 0121$ \\
\hline$\Gamma_{4} c_{1}$ & 14 & 224 & 132231 & $(2) 001$ \\
\hline$\Gamma_{4} c_{2}$ & 13 & 224 & 121121 & $(2) 0121$ \\
\hline$\Gamma_{4} c_{3}$ & 9 & 44 & 1344431 & - \\
\hline$\Gamma_{4} d$ & 10 & 344 & 111222111 & $(3) 023232$ \\
\hline$\Gamma_{5} a_{1}$ & 18 & 114 & 1221 & - \\
\hline$\Gamma_{5} a_{2}$ & 17 & 48 & $1,4,9,15$, & - \\
& & & 20,22, & \\
& & & 20,15, & \\
\hline$\Gamma_{6} a_{1}$ & 13 & 124 & 11011 & $(2) 011$ \\
\hline$\Gamma_{6} a_{2}$ & 11 & 28 & 134202431 & $(2) 00011$ \\
\hline$\Gamma_{7} a_{1}$ & 7 & 248 & 112211 & $(4) 0101$ \\
\hline$\Gamma_{7} a_{2}$ & 6 & see text & see text & see text \\
\hline$\Gamma_{7} a_{3}$ & 4 & 48 & 112202211 & $(4) 01001$ \\
\hline$\Gamma_{8} a_{1}$ & 5 & 12 & 11 & - \\
\hline$\Gamma_{8} a_{2}$ & 3 & 24 & 11011 & $(2) 011$ \\
\hline$\Gamma_{8} a_{3}$ & 2 & 4 & 1221 & - \\
\hline & & & & \\
\hline
\end{tabular}

\begin{tabular}{|l|l|l|l|}
\hline 1. Group & 8. Nilradical & $\begin{array}{c}\text { 9. Minimal } \\
\text { Prime Ideals }\end{array}$ & $\begin{array}{c}\text { 10. Essential Ideal } \\
\text { or Detecting Maximals }\end{array}$ \\
\hline$(5)$ & $(z)$ & $(z)$ & $(z)$ \\
\hline$(41)$ & $(y)$ & $(y)$ & $(z y)$ \\
\hline$(32)$ & $(z, y)$ & $(z, y)$ & $(z y)$ \\
\hline$(311)$ & $(x)$ & $(x)$ & $((z+y) z y x)$ \\
\hline$(221)$ & $(y, x)$ & $(y, x)$ & $(z y x)$ \\
\hline
\end{tabular}




\begin{tabular}{|l|l|l|l|}
\hline 1. Group & 8. Nilradical & $\begin{array}{c}\text { 9. Minimal } \\
\text { Prime Ideals }\end{array}$ & $\begin{array}{c}\text { 10. Essential Ideal } \\
\text { or Detecting Maximals }\end{array}$ \\
\hline$(2111)$ & $(w)$ & $(w)$ & $\begin{array}{c}(z y x(z+y)(z+x) \times \\
(y+x)(z+y+x) w)\end{array}$ \\
\hline$(11111)$ & $(0)$ & $(0)$ & $\begin{array}{c}\text { product of all } 31 \\
\text { elements of } H^{1}(G)\end{array}$ \\
\hline$\Gamma_{2} a_{1}$ & $(0)$ & $(y),(y+z)$ & $y, y+z$ \\
\hline$\Gamma_{2} a_{2}$ & $(x, w)$ & $(x, w)$ & $\left(x w^{2}\left(z^{2} y+z y^{2}\right)\right.$, \\
& & $x^{2}\left(z^{2} y+z y^{2}\right)^{2}$, \\
$\left.w^{2}\left(z^{2} y+z y^{2}\right)^{2}\right)$
\end{tabular}




\begin{tabular}{|c|c|c|c|}
\hline 1. Group & 8. Nilradical & $\begin{array}{c}\text { 9. Minimal } \\
\text { Prime Ideals } \\
\end{array}$ & $\begin{array}{l}\text { 10. Essential Ideal } \\
\text { or Detecting Maximals }\end{array}$ \\
\hline$\Gamma_{3} d_{2}$ & $(z, y)$ & $(z, y)$ & $(z y)$ \\
\hline$\Gamma_{3} e$ & $(y, x, v)$ & $\begin{array}{l}(z, y, x, v) \\
\quad(y, x, w, v)\end{array}$ & $z, y$ \\
\hline$\Gamma_{3} f$ & $(z, y, w)$ & $(z, y, w)$ & $(z y x)$ \\
\hline$\Gamma_{4} a_{1}$ & $(0)$ & $(z, x, u),(y)$ & $z, y$ \\
\hline$\Gamma_{4} a_{2}$ & $(0)$ & $\begin{array}{l}(z, y) \\
\quad(z, y+x) \\
(z+x, y) \\
(z+x \\
y+x)\end{array}$ & $z, z+x$ \\
\hline$\Gamma_{4} a_{3}$ & $(z, y, x)$ & $(z, y, x)$ & $(z y x)$ \\
\hline$\Gamma_{4} b_{1}$ & $(0)$ & $\begin{array}{l}(z, x) \\
\quad(z, x+y) \\
\quad(y, x)\end{array}$ & $z, y$ \\
\hline$\Gamma_{4} b_{2}$ & $(y, x)$ & $(y, x)$ & $z, y$ \\
\hline$\Gamma_{4} c_{1}$ & $(z)$ & $\begin{array}{r}(z, y, u) \\
\quad(z, x, v) \\
\end{array}$ & $y, x, y+x$ \\
\hline$\Gamma_{4} c_{2}$ & $(y)$ & $\begin{array}{l}(y, x) \\
\quad(y, x+z) \\
\end{array}$ & $y, y+z$ \\
\hline$\Gamma_{4} c_{3}$ & $(z, y, x, w, v)$ & $(z, y, x, w, v)$ & $\begin{array}{l}\left(z^{2} x, z y x, z y w, z x w\right. \\
\quad y x w)\end{array}$ \\
\hline$\Gamma_{4} d$ & $(y, x, w)$ & $(y, x, w)$ & $(z y x)$ \\
\hline$\Gamma_{5} a_{1}$ & (0) & $\begin{array}{c}(y, z),(y, w) \\
\quad(x, z) \\
(x, w) \\
(z+x \\
y+w) \\
(z+y \\
x+w)\end{array}$ & $z, y, z+y+x+w$ \\
\hline$\Gamma_{5} a_{2}$ & $\begin{array}{c}\left(y x+x^{2}\right. \\
z x+x^{2} \\
y w+w^{2} \\
\left.z w+w^{2}\right)\end{array}$ & $\begin{array}{l}z, x, w) \\
\quad(y, x, w) \\
(z+y \\
y+w, x) \\
(z+y \\
y+x, w) \\
(z+y \\
y+x \\
x+w) \\
\end{array}$ & $\begin{array}{l}z, y, z+y+x, z+y+w \\
z+y+x+w\end{array}$ \\
\hline
\end{tabular}




\begin{tabular}{|l|l|c|l|}
\hline 1. Group & 8. Nilradical & $\begin{array}{c}\text { 9. Minimal } \\
\text { Prime Ideals }\end{array}$ & $\begin{array}{c}\text { 10. Essential Ideal } \\
\text { or Detecting Maximals }\end{array}$ \\
\hline$\Gamma_{6} a_{1}$ & $(y x)$ & $(y),(z, x, w)$ & $z, y$ \\
\hline$\Gamma_{6} a_{2}$ & $(y, w$, & $(y, x, w, v)$, & $\left(z y^{3}\right)$ \\
& $x^{2}+x z$, & $(z+x, y$, & \\
& $v x+v z)$ & $w)$ & \\
\hline$\Gamma_{7} a_{1}$ & $(y)$ & $(y, v+x, u$, & $z, y, z+y$ \\
& & $\begin{array}{c}\left.w+x+z^{2}\right), \\
(z, y)\end{array}$ & \\
& & $(y, w, u, t)$, & $z, y$ \\
& $(y)$ & $\left(y, w+z^{2}\right.$, & \\
& & $t+z z$, & \\
& & $(y, w, u, t)$, & $\left(z^{3}+z^{2} y, z^{2} w+z y w\right)$ \\
& & $\left(y, w+z^{2}\right.$, & \\
& & $u+x z$, & \\
& & $t+z v)$ & \\
\hline$\Gamma_{7} a_{3}$ & $(z, y, w, v, u$, & $(y),(y+z)$ & $y, y+z$ \\
\hline$\Gamma_{8} a_{1}$ & $(0)$ & $(y)$ & $y, y+z$ \\
\hline$\Gamma_{8} a_{2}$ & $(y)$ & $\left(z^{2}, z y\right)$ \\
\hline$\Gamma_{8} a_{3}$ & $(z, y)$ & $(z, y)$ & \\
\hline
\end{tabular}

\begin{tabular}{|l|l|l|l|l|}
\hline 1. Group & $\begin{array}{c}\text { 11. Minimal } \\
\text { Detectors }\end{array}$ & $\begin{array}{c}\text { 12. Elemen. } \\
\text { Subgps. }\end{array}$ & $\begin{array}{c}\text { 13. Refs. in } \\
\text { this paper }\end{array}$ & $\begin{array}{c}\text { 14. Prev. } \\
\text { Papers }\end{array}$ \\
\hline$(5)$ & - & 1I & - & - \\
\hline$(41)$ & - & 2I & - & {$[12]$} \\
\hline$(32)$ & - & 2I & 2.1 & {$[12]$} \\
\hline$(311)$ & - & 3I & - & - \\
\hline$(221)$ & - & 3I & - & - \\
\hline$(2111)$ & - & $4 \mathrm{I}$ & - & {$[10]$} \\
\hline$(11111)$ & - & 5I & 2.5 & {$[10]$} \\
\hline$\Gamma_{2} a_{1}$ & $2\left(1^{4}\right)$ & $4 \mathrm{IV} 2$ & 2.5 & {$[6],[10]$} \\
\hline$\Gamma_{2} a_{2}$ & - & $3 \mathrm{I}$ & - & {$[2],[10]$} \\
\hline$\Gamma_{2} b$ & $3\left(21^{2}\right)$ & 3IV3 & 1.8 & {$[10]$} \\
\hline$\Gamma_{2} c_{1}$ & $1^{4}, 21^{2}$ & $4 \mathrm{II}$ & 2.5 & - \\
\hline
\end{tabular}




\begin{tabular}{|l|l|l|l|l|}
\hline 1. Group & $\begin{array}{c}11 . \text { Minimal } \\
\text { Detectors }\end{array}$ & $\begin{array}{c}\text { 12. Elemen. } \\
\text { Subgps. }\end{array}$ & $\begin{array}{c}\text { 13. Refs. in } \\
\text { this paper }\end{array}$ & $\begin{array}{c}14 . \text { Prev. } \\
\text { Papers }\end{array}$ \\
\hline$\Gamma_{2} c_{2}$ & - & $3 \mathrm{I}$ & - & {$[12]$} \\
\hline$\Gamma_{2} d$ & - & $3 \mathrm{II}$ & 2.5 & {$[12]$} \\
\hline$\Gamma_{2} e_{1}$ & $2\left(21^{2}\right)$ & $3 \mathrm{IV} 2$ & 1.8 & {$[6]$} \\
\hline$\Gamma_{2} e_{2}$ & - & $2 \mathrm{I}$ & 1.8 & {$[2]$} \\
\hline$\Gamma_{2} f$ & - & $3 \mathrm{II}$ & $1.6,1.8,2.5$ & - \\
\hline$\Gamma_{2} g$ & $3(31)$ & $2 \mathrm{IV} 3$ & 1.8 & - \\
\hline$\Gamma_{2} h$ & - & $3 \mathrm{I}$ & 1.8 & - \\
\hline$\Gamma_{2} i$ & - & $2 \mathrm{I}$ & 2.1 & {$[12]$} \\
\hline$\Gamma_{2} j_{1}$ & $31,21^{2}$ & $3 \mathrm{II}$ & 2.5 & - \\
\hline$\Gamma_{2} j_{2}$ & - & $2 \mathrm{I}$ & 2.1 & {$[12]$} \\
\hline$\Gamma_{2} k$ & - & $2 \mathrm{II}$ & 2.5 & {$[12]$} \\
\hline$\Gamma_{3} a_{1}$ & $2\left(1^{3}\right)$ & $3 \mathrm{~V} 22$ & 2.5 & {$[6]$} \\
\hline$\Gamma_{3} a_{2}$ & $1^{3}, Q_{8} \times \mathbf{Z}_{2}$ & $3 \mathrm{III}$ & - & {$[4]$} \\
\hline$\Gamma_{3} a_{3}$ & - & $2 \mathrm{I}$ & - & {$[2]$} \\
\hline$\Gamma_{3} b$ & $2(21),(31)$ & $2 \mathrm{~V} 122$ & 1.8 & - \\
\hline$\Gamma_{3} c_{1}$ & $1^{3}, \mathbf{Z}_{4} \cdot \mathbf{Z}_{4}$ & $3 \mathrm{III}$ & - & - \\
\hline$\Gamma_{3} c_{2}$ & - & $2 \mathrm{I}$ & $1.8,1.9$ & - \\
\hline$\Gamma_{3} d_{1}$ & - & $2 \mathrm{I}$ & 2.1 & {$[12]$} \\
\hline$\Gamma_{3} d_{2}$ & - & $2 \mathrm{I}$ & $1.7,2.1$ & {$[12]$} \\
\hline$\Gamma_{3} e$ & $(12), 2^{2}$ & $2 \mathrm{~V} 12$ & 1.8 & - \\
\hline$\Gamma_{3} f$ & - & $2 \mathrm{II}$ & 2.5 & {$[12]$} \\
\hline$\Gamma_{4} a_{1}$ & $1^{3}, 1^{3}$ & $4 \mathrm{VI}$ & $2.3,2.4$ & {$[11]$} \\
\hline & & & $2.5,2.6$ & \\
\hline$\Gamma_{4} a_{2}$ & $4\left(1^{3}\right)$ & $3 \mathrm{IV} 4$ & 2.5 & - \\
\hline$\Gamma_{4} a_{3}$ & - & $2 \mathrm{I}$ & - & - \\
\hline$\Gamma_{4} b_{1}$ & $2\left(1^{3}\right),\left(21^{2}\right)$ & $3 \mathrm{IV} 3$ & - & - \\
\hline$\Gamma_{4} b_{2}$ & $21^{2}, Q_{8} \times \mathbf{Z}_{2}$ & $3 \mathrm{II}$ & 2.5 & - \\
\hline$\Gamma_{4} c_{1}$ & $(3)(21)$, & $3 \mathrm{IV} 2$ & 1.8 & - \\
\hline$\Gamma_{4} c_{2}$ & $22,2\left(1^{3}\right)$ & $3 \mathrm{IV} 2$ & - & - \\
\hline$\Gamma_{4} c_{3}$ & - & $2 \mathrm{I}$ & $1.8,1.9$ & - \\
\hline$\Gamma_{4} d$ & - & $3 \mathrm{II}$ & $1.6,1.8,1.9$ & - \\
\hline$\Gamma_{5} a_{1}$ & $6\left(1^{3}\right)$ & $3 \mathrm{IV} 6$ & 2.5 & {$[10]$} \\
\hline & & & & \\
\hline
\end{tabular}




\begin{tabular}{|l|l|l|l|l|}
\hline 1 Group & $\begin{array}{c}\text { 11. Minimal } \\
\text { Detectors }\end{array}$ & $\begin{array}{c}\text { 12. Elemen. } \\
\text { Subgps. }\end{array}$ & $\begin{array}{c}\text { 13. Refs. in } \\
\text { this paper }\end{array}$ & $\begin{array}{c}\text { 14. Prev. } \\
\text { Papers }\end{array}$ \\
\hline$\Gamma_{5} a_{2}$ & $\begin{array}{c}3\left(Q_{8} \times \mathbf{Z}_{2}\right), \\
3(21)\end{array}$ & $2 \mathrm{IV} 5$ & 1.9 & {$[10]$} \\
\hline$\Gamma_{6} a_{1}$ & $1^{3}, 2(21)$ & $3 \mathrm{VI}$ & 2.6 & - \\
\hline$\Gamma_{6} a_{2}$ & - & $2 \mathrm{~V} 12$ & $1.8,2.3$ & - \\
\hline$\Gamma_{7} a_{1}$ & $2(21), 2\left(1^{3}\right)$ & $3 \mathrm{IV} 2$ & 1.6 & - \\
\hline$\Gamma_{7} a_{2}$ & $2\left(1^{3}\right), Q D_{16}$ & $3 \mathrm{IV} 2$ & 2.3 & - \\
\hline$\Gamma_{7} a_{3}$ & - & $2 \mathrm{II}$ & $1.6,1.8,2.1$ & {$[13]$} \\
\hline$\Gamma_{8} a_{1}$ & $2\left(1^{2}\right)$ & $2 \mathrm{~V} 44$ & - & {$[6]$} \\
\hline$\Gamma_{8} a_{2}$ & $\left(1^{2}\right), Q_{16}$ & $2 \mathrm{III}$ & - & {$[4],[12]$} \\
\hline$\Gamma_{8} a_{3}$ & - & $1 \mathrm{I}$ & - & {$[2]$} \\
\hline
\end{tabular}

Department of Mathematics

Northern Illinois University

DeKalb, Illinois 60115

1. M. F. AtiYAH, "Characters and cohomology of finite groups," Publ. Math. Inst. Hautes Etudes Sci., v. 9, 1961, pp. 23-64.

2. H. Cartan \& S. Eilenberg, Homological Algebra, Princeton Univ. Press, Princeton, NJ, 1956.

3. J. DUflot, "Depth and equivariant cohomology," Comment. Math. Helv., v. 56, 1981, pp. 627-637.

4. L. EVENS \& S. PRIDDY, (unpublished).

5. M. FESHBACH \& S. PRIDDY, "Stable splittings associated with Chevalley groups," $J$. Pure Appl. Algebra. (To appear.)

6. Z. FIEDOROWICZ \& S. PRIDDY, Homology of Classical Groups Over Finite Fields, Lecture Notes in Math., vol. 674, Springer-Verlag, Berlin and New York, 1978.

7. M. HALL \& J. K. SENIOR, The Groups of Order $2^{n}(n \leq 6)$, Macmillan, New York, 1964. 1971.

8. P. Hilton \& U. Stammbach, A Course in Homological Algebra, Springer, New York,

9. D. QUILLEN, "The spectrum of an equivariant cohomology ring, I and II," Ann. of Math., v. 94,1971, pp. $549-602$.

10. D. QUILLEN, "The mod-2 cohomology of extraspecial 2-groups and the spinor groups," Math. Ann., v. 194, 1971, pp. 197-212.

11. D. RUSIN, The Cohomology of Groups Generated by Involutions, Thesis, Univ. of Chicago, 1984.

12. D. RUSIN, "The mod-2 cohomology of metacyclic 2-groups," J. Pure Appl. Algebra, v. 44, 1987, pp. 315-327.

13. D. RUSIN, "The kernel of the inflation map in group cohomology," J. Pure Appl. Algebra. (To appear.)

14. D. RUSIN, "Kernels of the restriction map in group cohomology," J. Pure Appl. Algebra. (To appear.) 